\title{
New Transmission Scheme Based on Space-Time Code for Massive MIMO
}

\author{
Xinji Tian, Wenjie Jia, Haotian Zhang \\ School of Physics and Electronic Information Engineering, Henan Polytechnic University, Jiaozuo, China \\ Email: 718213108@qq.com
}

How to cite this paper: Tian, X.J., Jia, W.J and Zhang, H.T. (2017) New Transmission Scheme Based on Space-Time Code for Massive MIMO. Open Access Library Journal, 4: e3712.

https://doi.org/10.4236/oalib.1103712

Received: June 6, 2017

Accepted: June 26, 2017

Published: June 29, 2017

Copyright $\odot 2017$ by authors and Open Access Library Inc.

This work is licensed under the Creative Commons Attribution International License (CC BY 4.0).

http://creativecommons.org/licenses/by/4.0/

\section{c) (i) Open Access}

\begin{abstract}
New transmission scheme based on space-time code is proposed for massive MIMO system, which consists of $K$ users and a base station. Each user is equipped with four antennas and two of them are selected to send space-time codeword with Alamouti coded by using the method of generalized spatial modulation. The base station estimates the index of transmit antennas used by each user at first, and then uses an iterative method to separate each spacetime codeword and decode modulated signals of each code-word. Compared with the existing scheme for the same scene, the proposed scheme reduces the complexity of decoding, while keeping the same transmission rate. Simulation results demonstrate that the proposed scheme outperforms the existing scheme in the same scene when the base station is equipped with large amount of antennas.
\end{abstract}

\section{Subject Areas}

Information and Communication Theory and Algorithms

\section{Keywords}

Massive MIMO Systems, Space-Time Code, Decoding Complexity, Generalized Spatial Modulation

\section{Introduction}

Transmission rate and spectrum efficiency are particularly important for multicell communication. Massive MIMO technology have the ability to improve spectrum efficiency, cut down transmission power, reduce intra cell interference and inter-cell interference of the system without increasing spectrum resources [1]. So, it becomes one of the breakthrough technologies in the future [2].

In massive MIMO system, the base station is equipped with hundreds of antennas. $K$ users transmit modulated signals simultaneously, or transmit the sig- 
nals after precoding at the same time in a single cell uplink system [3]. If all of the users are equipped with $n_{t}$ antennas, there will be $n_{t}$ modulation symbols sent by each user per time slot [4] [5]. Generalized spatial modulation is studied in [6], in which not only transmit antennas send symbols, but also different antenna combinations are mapped into several bits. Thus, the transmission efficiency is improved. However, the decoding complexity of Maximum Likelihood (ML) decoding, Message Passing (MP) decoding and Channel Hardening Exploiting Message Passing (CHEMP) in [6] is too high.

In order to reduce the decoding complexity, the transmission based on spacetime coding is proposed in single-cell massive MIMO uplink system. All of the users are equipped with 4 antennas and generalized spatial modulation is used at each user. According to the information sequence, two of the antennas are selected to transmit the space-time code which has Alamouti structure. Firstly, the serial number of the transmit antenna used at each user are estimated at the base station. Then, each space-time codeword is separated using the orthogonality of Alamouti code. Finally, modulation symbols in each codeword are detected. The ML decoding complexity of the proposed scheme is proportional to the square of the modulation order. The simulation results show that the reliability of the proposed scheme is better than [6].

\section{System Model}

The system model of the proposed scheme is shown in Figure 1, which contains $K$ users and a base station. All users are equipped with 4 antennas and the base station is equipped with $N(N \geq 4 K)$ antennas. Generalized spatial modulation proposed in [6] is adopted. Each user selects two antennas to transmit signals according to the information sequence. When the information sequences are 00 , 01,10 and 11 , the corresponding antenna combinations are $(1,2),(1,3),(1,4)$ and $(2,3)$, respectively.

Rate-2 space-time code proposed in [7] is used at user $k, k=1,2, \cdots, K$. The codeword at user $k$, denoted by $\boldsymbol{X}_{k}^{\prime}$, is expressed as

$$
\boldsymbol{X}_{k}^{\prime}=\left[\begin{array}{l}
\boldsymbol{x}_{1} \\
\boldsymbol{x}_{2}
\end{array}\right]=\left[\begin{array}{cc}
x_{k, 1}^{\prime} & -x_{k, 2}^{\prime}{ }^{*} \\
x_{k, 2}^{\prime} & x_{k, 1}^{\prime}{ }^{*}
\end{array}\right]=\left[\begin{array}{cc}
x_{k, 1}+e^{j \theta} x_{k, 2} & -x_{k, 3}{ }^{*}-e^{-j \theta} x_{k, 4} \\
x_{k, 3}^{k}+e^{j \theta} x_{k, 4} & x_{k, 1}{ }^{*}+e^{-j \theta} x_{k, 2}{ }^{*}
\end{array}\right]
$$

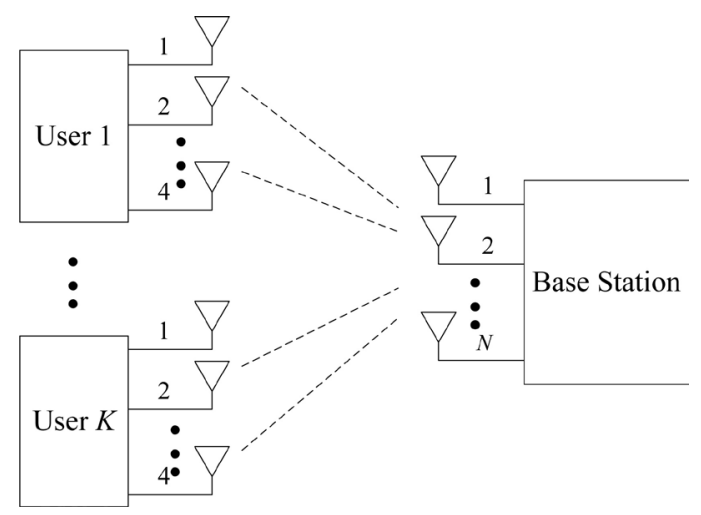

Figure 1. System model of the proposed scheme. 
where, $x_{k, \lambda}$ represents the modulation symbols, $\lambda=1,2,3,4, j=\sqrt{-1},(\cdot)^{*}$ represents conjugate. The value of $\theta$ makes the elements of $\boldsymbol{X}_{k}^{\prime}$ nonzero.

Assume that $\left(\mu_{k}, v_{k}\right)$ are the antenna numbers selected by user $k$, $\mu_{k} \neq v_{k} \in\{1,2,3,4\}, \quad \mu_{k}<v_{k}$. Then the antenna $\mu_{k}$ at user $k$ sends $\boldsymbol{x}_{1}$, the antenna $v_{k}$ sends $\boldsymbol{x}_{2}$ and the other antennas send $\left[\begin{array}{ll}0 & 0\end{array}\right]$. Use $\boldsymbol{X}_{k}$ to represent the codeword send by user $k$. The dimension of $\boldsymbol{X}_{k}$ is $4 \times 2$. The elements of $\boldsymbol{X}_{k}$ in the row of $\mu_{k}$ and $v_{k}$ are $\boldsymbol{x}_{1}$ and $\boldsymbol{x}_{2}$ respectively, and the elements in other rows are zero.

The received signals at the base station, denoted by $\boldsymbol{Y}$, can be expressed as

$$
\begin{aligned}
& \boldsymbol{Y}=\boldsymbol{H}_{1} \boldsymbol{X}_{1}+\boldsymbol{H}_{2} \boldsymbol{X}_{2}+\cdots+\boldsymbol{H}_{K} \boldsymbol{X}_{K}+\boldsymbol{N} \\
& =\underbrace{\left[\begin{array}{cccccccccc}
h_{1,1}^{1} & \cdots & h_{1,4}^{1} & h_{1,1}^{2} & \cdots & h_{1,4}^{2} & \cdots & h_{1,1}^{K} & \cdots & h_{1,4}^{K} \\
\vdots & \ddots & \vdots & \vdots & \ddots & \vdots & \ddots & \vdots & \ddots & \vdots \\
h_{N, 1}^{1} & \cdots & h_{N, 4}^{1} & h_{N, 1}^{2} & \cdots & h_{N, 4}^{2} & \cdots & h_{N, 1}^{K} & \cdots & h_{N, 4}^{K}
\end{array}\right]}_{\boldsymbol{H}}\left[\begin{array}{cc}
\boldsymbol{x}_{1,1} & \boldsymbol{x}_{1,2} \\
\vdots & \vdots \\
\boldsymbol{x}_{K, 1} & \boldsymbol{x}_{K, 2}
\end{array}\right]+\underbrace{\left[\begin{array}{cc}
n_{1,1} & n_{1,2} \\
\vdots & \vdots \\
n_{N, 1} & n_{N, 2}
\end{array}\right]}_{\boldsymbol{N}}
\end{aligned}
$$

where, dimension of $\boldsymbol{Y}$ and $N$ are both $N \times 2 . N$ represents the complex Gauss noise matrix with zero mean and variance of $\sigma^{2} . \boldsymbol{H}_{k}$, with dimension $N \times 4$, represents the channel fading coefficient from user $k$ to the base station. The dimension of $\boldsymbol{x}_{k, 1}$ and $\boldsymbol{x}_{k, 2}$ are both $4 \times 1$. The $\mu_{k}$-th and $v_{k}$-th elements of $x_{k, 1}$ are $x_{k, 1}^{\prime}$ and $x_{k, 2}^{\prime}$, respectively. The $\mu_{k}$-th element and $v_{k}$-th element of $\boldsymbol{x}_{k, 2}$ are $x_{k, 1}^{\prime}$ and $x_{k, 2}^{\prime}$, respectively. The other elements of $\boldsymbol{x}_{k, 1}$ and $\boldsymbol{x}_{k, 2}$ are zeros.

\section{Detection}

The detection at the base station can be divided into two steps. Firstly, the serial number of the transmit antennas at each user is estimated at the base station according to the received signals and the channel state information (CSI). Then, each codeword is separated and each element of the codeword is estimated using iterative method at the base station.

Use $\boldsymbol{y}_{1}^{\prime}$ to represent the first column of $\boldsymbol{Y}$. Let $\boldsymbol{g}=\operatorname{pinv}(\boldsymbol{H}) \boldsymbol{y}_{1}^{\prime}$, which $\operatorname{pinv}(\cdot)$ represents pseudo inverse. $\boldsymbol{g}$ is a vector with a dimension of $4 K \times 1$. Use $\overline{\boldsymbol{g}}_{k}$ to represent the vector with a dimension of $4 \times 1$, which consists of the $4(k-1)+1-t h$ element to $4 k$-th emelent of $\boldsymbol{g}, k=1,2, \cdots, K$. According to the estimation method of transmitting antennas' number in (2) and [8], the serial number of the transmit antennas for user $k$ obtained at the base station can be expressed as

$$
\left(\mu_{k 0}, v_{k 0}\right)=\underset{\substack{g_{\mu^{\prime}}, g_{v_{k}^{\prime}} \in \bar{g}_{k} \\ \mu_{k}^{\prime} \neq v_{k}^{\prime}}}{\arg \max }\left(\left\|g_{\mu_{k}^{\prime}}\right\|+\left\|g_{v_{k}^{\prime}}\right\|\right)
$$

where, $\mu_{k 0} \neq v_{k 0} \in\{1,2,3,4\}, \quad g_{\mu_{k}^{\prime}}$ and $g_{v_{k}^{\prime}}$ represents the $\mu^{\prime}$-th and $v^{\prime}-t h$ element of $\overline{\boldsymbol{g}}_{k} \cdot\|\cdot\|$ represents norm.

After the serial number of transmit antenna is estimated, the zero elements in (2) can be removed. Then, (2) can be equivalently expressed as

$$
\boldsymbol{Y}=\boldsymbol{H}_{1}^{\prime} \boldsymbol{X}_{1}^{\prime}+\boldsymbol{H}_{2}^{\prime} \boldsymbol{X}_{2}^{\prime}+\cdots+\boldsymbol{H}_{K}^{\prime} \boldsymbol{X}_{K}^{\prime}+\boldsymbol{N}
$$


where, $\boldsymbol{X}_{k}^{\prime}$ represents a $2 \times 2$ matrix after removing the zero elements in $\boldsymbol{X}_{k}$, which is expressed as (1), $k=1,2, \cdots, K . \quad \boldsymbol{H}_{k}^{\prime}$ represents a $N \times 2$ matrix, which consists of $\mu_{k}$-th column and $v_{k}$-th column of $\boldsymbol{H}_{k}$.

Since $\boldsymbol{X}_{k}^{\prime}$ has Alamouti structure, (4) can be expressed as

$$
\left[\begin{array}{c}
\boldsymbol{y}_{1} \\
\boldsymbol{y}_{2} \\
\vdots \\
\boldsymbol{y}_{N}
\end{array}\right]=\left[\begin{array}{cccc}
\boldsymbol{H}_{1}^{1} & \boldsymbol{H}_{1}^{2} & \cdots & \boldsymbol{H}_{1}^{K} \\
\vdots & \vdots & \ddots & \vdots \\
\boldsymbol{H}_{N}^{1} & \boldsymbol{H}_{N}^{2} & \cdots & \boldsymbol{H}_{N}^{K}
\end{array}\right]\left[\begin{array}{c}
\boldsymbol{s}_{1} \\
\vdots \\
\boldsymbol{s}_{K}
\end{array}\right]+\left[\begin{array}{c}
\boldsymbol{n}_{1} \\
\boldsymbol{n}_{2} \\
\vdots \\
\boldsymbol{n}_{N}
\end{array}\right]
$$

where $\boldsymbol{H}_{i}^{k}=\left[\begin{array}{cc}h_{i, 1}^{k} & h_{i, 2}^{k} \\ h_{i, 2}^{k *} & -h_{i, 1}^{k *}\end{array}\right], \quad \boldsymbol{n}_{i}=\left[\begin{array}{ll}n_{i, 1} & n_{i, 2}{ }^{*}\end{array}\right]^{\mathrm{T}}, \quad y_{i, j}$ represents the element of $\boldsymbol{Y}$ in row $i$ and column $j, j=1,2 .(\cdot)^{\mathrm{T}}$ represents the transpose.

According to the method of separating Alamouti codewords in [9] and [10], (5) can be decomposed into $\Lambda=\lfloor N / K\rfloor$ sub-equations, which $\lfloor\cdot\rfloor$ represents round down operation. Each sub-equation can be expressed as

$$
\begin{aligned}
& \underbrace{\left[\begin{array}{c}
\boldsymbol{y}_{1} \\
\vdots \\
\boldsymbol{y}_{K}
\end{array}\right]}_{\bar{y}_{1}}=\underbrace{\left[\begin{array}{cccc}
\boldsymbol{H}_{1}^{1} & \boldsymbol{H}_{1}^{2} & \cdots & \boldsymbol{H}_{1}^{K} \\
\vdots & \vdots & \ddots & \vdots \\
\boldsymbol{H}_{K}^{1} & \boldsymbol{H}_{K}^{2} & \cdots & \boldsymbol{H}_{K}^{K}
\end{array}\right]}_{\overline{\boldsymbol{H}}_{1}}\left[\begin{array}{c}
\boldsymbol{s}_{1} \\
\vdots \\
\boldsymbol{s}_{K}
\end{array}\right]+\underbrace{\left[\begin{array}{c}
\boldsymbol{n}_{1} \\
\vdots \\
\boldsymbol{n}_{K}
\end{array}\right]}_{\overline{\boldsymbol{n}}_{1}} \\
& \underbrace{\left[\begin{array}{c}
\boldsymbol{y}_{(\Lambda-1) K+1} \\
\vdots \\
\boldsymbol{y}_{\Lambda K}
\end{array}\right]}_{\overline{\boldsymbol{y}}_{\Lambda}}=\underbrace{\left[\begin{array}{cccc}
\boldsymbol{H}_{(\Lambda-1) K+1}^{1} & \boldsymbol{H}_{(\Lambda-1) K+1}^{2} & \ldots & \boldsymbol{H}_{(\Lambda-1) K+1}^{K} \\
\vdots & \vdots & \ddots & \vdots \\
\boldsymbol{H}_{\Lambda K}^{1} & \boldsymbol{H}_{\Lambda K}^{2} & \cdots & \boldsymbol{H}_{\Lambda K}^{K}
\end{array}\right]}_{\overline{\boldsymbol{H}}_{\Lambda}}\left[\begin{array}{c}
\boldsymbol{s}_{1} \\
\vdots \\
\boldsymbol{s}_{K}
\end{array}\right]+\underbrace{\left[\begin{array}{c}
\boldsymbol{n}_{(\Lambda-1) K+1} \\
\vdots \\
\boldsymbol{n}_{\Lambda K}
\end{array}\right]}_{\overline{\boldsymbol{n}}_{\Lambda}}
\end{aligned}
$$

Let

$$
\begin{aligned}
& \boldsymbol{G}_{\ell, j}(l)=\left[\begin{array}{ll}
\boldsymbol{I}_{2} & \boldsymbol{F}_{\ell, j}{ }^{H}(l)
\end{array}\right]^{H} \\
& =[\boldsymbol{I}_{2} \underbrace{-\frac{\overline{\boldsymbol{H}}_{\ell, j}^{K-l+1}(l-1) \overline{\boldsymbol{H}}_{\ell, K-l+1}^{K-l+1} H(l-1)}{\left\|\overline{\boldsymbol{H}}_{\ell, K-l+1}^{K-l+1}(l-1)\right\|^{2}}}_{\boldsymbol{F}_{\ell, j}(l)}], \\
& l \in\{1, \cdots, K-1\}, \quad j \in\{1, \cdots, K-l \mid l\}
\end{aligned}
$$

where, $(\cdot)^{H}$ represents conjugate transpose, $\boldsymbol{I}_{2}$ represents a unit matrix with a dimension of $2 \times 2$. From (4)-(8) in [10], codewords can be separated gradually by $(K-1)$ times iteration. The matrix, vector and element with subscript $\ell$ belong to the $\ell$-th sub-equation after decomposition, $\ell=1, \cdots, \Lambda$.

From (25) in [10], we can find that, the $\ell$-th sub-formula from (6) can be expressed as (8) after $(K-1)$ times iterative,

$$
\hat{\boldsymbol{G}}_{\ell}{ }_{\ell} \overline{\boldsymbol{y}}_{\ell}=\hat{\boldsymbol{G}}_{\ell}{ }_{\ell}\left[\begin{array}{c}
\boldsymbol{H}_{(\ell-1) K+1}^{1} \\
\vdots \\
\boldsymbol{H}_{\ell K}^{1}
\end{array}\right] \boldsymbol{s}_{1}+\hat{\boldsymbol{G}}_{\ell}{ }^{H} \overline{\boldsymbol{n}}_{\ell}=\underbrace{\hat{\boldsymbol{G}}_{\ell}^{H}\left[\overline{\boldsymbol{h}}_{\ell, 1}\right.}_{\tilde{\boldsymbol{H}}_{\ell}} \overline{\boldsymbol{h}}_{\ell, 2}] \boldsymbol{s}_{1}+\hat{\boldsymbol{G}}_{\ell}{ }^{H} \overline{\boldsymbol{n}}_{\ell}
$$


where, $\hat{\boldsymbol{G}}_{\ell}=\left[\begin{array}{c}\boldsymbol{A}_{\ell, 1} \\ \boldsymbol{A}_{\ell, 2} \\ \boldsymbol{A}_{\ell, 3} \\ \vdots \\ \boldsymbol{A}_{\ell, K}\end{array}\right]=\left[\begin{array}{c}\boldsymbol{I}_{2} \\ \boldsymbol{F}_{\ell, 1}(K-1) \\ \boldsymbol{F}_{\ell, 1}(K-2)+\varphi_{\ell}^{(1)}\left(\boldsymbol{A}_{\ell, 2}\right) \boldsymbol{F}_{\ell, 1}(K-1) \\ \vdots \\ \sum_{l=1}^{K-1} \varphi_{\ell}^{(K-l)}\left(\boldsymbol{A}_{\ell, l}\right) \boldsymbol{F}_{\ell, 1}(l)\end{array}\right], \varphi_{\ell}^{(n)}\left(\boldsymbol{A}_{\ell, k}\right)$

represents a function replace all of $\boldsymbol{F}_{\ell, \psi}(l)$ in $\boldsymbol{A}_{\ell, k}$ to $\boldsymbol{F}_{\ell, \psi+n}(l-n)$, $n \in\{1, \cdots, K-1\}, \psi \in\{1, \cdots, K-1\} . \varphi_{\ell}^{(n)}\left(\boldsymbol{I}_{2}\right)=\boldsymbol{I}_{2}, \quad \boldsymbol{A}_{\ell, k}$ is a matrix with dimension $2 \times 2, \overline{\boldsymbol{h}}_{\ell, 1}$ and $\overline{\boldsymbol{h}}_{\ell, 2}$ represent the 1-th column and 2-th column of $\left[\begin{array}{llll}\left(\boldsymbol{H}_{(\ell-1) K+1}^{1}\right)^{H} & \left(\boldsymbol{H}_{(\ell-1) K+2}^{1}\right)^{H} & \cdots & \left(\boldsymbol{H}_{\ell K}^{1}\right)^{H}\end{array}\right]^{H}$ respectively.

Let $\quad \tilde{\boldsymbol{H}}_{\ell}=\hat{\boldsymbol{G}}_{\ell}{ }^{H}\left[\begin{array}{ll}\overline{\boldsymbol{h}}_{\ell, 1} & \overline{\boldsymbol{h}}_{\ell, 2}\end{array}\right]$, and multiply both sides of (8) by $\tilde{\boldsymbol{H}}_{\ell}{ }^{H}$ to get

$$
\tilde{\boldsymbol{H}}_{\ell}{ }^{H} \hat{\boldsymbol{G}}_{\ell}{ }^{H} \overline{\boldsymbol{y}}_{\ell}=\tilde{\boldsymbol{H}}_{\ell}{ }^{H} \tilde{\boldsymbol{H}}_{\ell} \boldsymbol{s}_{1}+\tilde{\boldsymbol{H}}_{\ell}{ }^{H} \hat{\boldsymbol{G}}_{\ell}{ }^{H} \overline{\boldsymbol{n}}_{\ell}
$$

Since the matrix with Alamouti structure is closed for addition, multiplication, and conjugate transpose operations, $\tilde{\boldsymbol{H}}_{\ell}$ has Alamouti structure, which result in $\tilde{\boldsymbol{H}}_{\ell}{ }^{H} \tilde{\boldsymbol{H}}_{\ell}=\left(\overline{\boldsymbol{h}}_{1,1}{ }^{H} \hat{\boldsymbol{G}}_{1} \hat{\boldsymbol{G}}_{1}{ }^{H} \overline{\boldsymbol{h}}_{1,1}+\cdots+\overline{\boldsymbol{h}}_{\Lambda, 1}{ }^{H} \hat{\boldsymbol{G}}_{\Lambda} \hat{\boldsymbol{G}}_{\Lambda}{ }^{H} \overline{\boldsymbol{h}}_{\Lambda, 1}\right) \boldsymbol{I}_{2}$. Use $\tilde{r}_{1}$ to represent the first element of $\tilde{\boldsymbol{H}}_{\ell}{ }^{H} \hat{\boldsymbol{G}}_{\ell}{ }^{H} \overline{\boldsymbol{y}}_{\ell}$, then (10) can be obtained by (6)-(9).

$$
\begin{aligned}
\tilde{r}_{1}= & \left(\overline{\boldsymbol{h}}_{1,1}{ }^{H} \hat{\boldsymbol{G}}_{1} \hat{\boldsymbol{G}}_{1}{ }^{H} \overline{\boldsymbol{h}}_{1,1}+\cdots+\overline{\boldsymbol{h}}_{\Lambda, 1}{ }^{H} \hat{\boldsymbol{G}}_{\Lambda} \hat{\boldsymbol{G}}_{\Lambda}{ }^{H} \overline{\boldsymbol{h}}_{\Lambda, 1}\right) x_{1,1}^{\prime} \\
& +\left(\overline{\boldsymbol{h}}_{1,1}{ }^{H} \hat{\boldsymbol{G}}_{1} \hat{\boldsymbol{G}}_{1}{ }^{H} \overline{\boldsymbol{n}}_{1}+\cdots+\overline{\boldsymbol{h}}_{\Lambda, 1}{ }^{H} \hat{\boldsymbol{G}}_{\Lambda} \hat{\boldsymbol{G}}_{\Lambda}{ }^{H} \overline{\boldsymbol{n}}_{\Lambda}\right)
\end{aligned}
$$

The base station can get $x_{1,1}^{\prime}$ from $\tilde{r}_{1}$ directly. The detection of the other modulation symbols is similar. Since $\boldsymbol{X}_{k}^{\prime}$ has Alamouti structure and each codeword consists of two modulation symbols, the complexity of ML decoding is proportional to the square of the modulation order.

\section{Bit Error Rate Analysis}

Let $P_{e 1}$ and $P_{e 2}$ represents the error probability of estimating the number of transmit antennas and the symbol error rate (SER) of the second step. Then the SER of the system can be expressed as

$$
P_{e}=1-\left(1-P_{e 1}\right)\left(1-P_{e 2}\right)
$$

We deduce $P_{e 2}$ firstly. Assume the mathematical expectation of the modulation signal is $P_{1}$. The signal-noise ratio (SNR) during the process of decoding $x_{1,1}^{\prime}$, denoted by $\rho$, can be obtained from (10).

$$
\rho=\frac{P_{1}}{\sigma^{2}} \frac{\left(\overline{\boldsymbol{h}}_{1,1}{ }^{H} \hat{\boldsymbol{G}}_{1,1} \hat{\boldsymbol{G}}_{1}{ }^{H} \overline{\boldsymbol{G}}_{1,1} \hat{\boldsymbol{G}}_{1}{ }^{\mathrm{H}}{ }^{\mathrm{G}} \hat{\boldsymbol{G}}_{1} \hat{\boldsymbol{G}}_{1}{ }^{H} \overline{\boldsymbol{h}}_{1,1}+\cdots+\left(\overline{\boldsymbol{h}}_{\Lambda, 1}{ }^{H} \hat{\boldsymbol{G}}_{\Lambda} \hat{\boldsymbol{G}}_{\Lambda}{ }^{H} \overline{\boldsymbol{h}}_{\Lambda, 1}\right)^{2}{ }^{H} \hat{\boldsymbol{G}}_{\Lambda} \hat{\boldsymbol{G}}_{\Lambda}{ }^{H} \hat{\boldsymbol{G}}_{\Lambda} \hat{\boldsymbol{G}}_{\Lambda}{ }^{H} \overline{\boldsymbol{h}}_{\Lambda, 1}\right.}{}
$$

According to the estimation method of SER in [10] and [11], the probability of estimating $x_{1,1}^{\prime}$ as $\hat{x}_{1,1}^{\prime}$, denoted by $P_{e 2}$, can be expressed as

$$
\begin{aligned}
P_{e 2} & =P\left(x_{1,1}^{\prime} \rightarrow \hat{x}_{1,1}^{\prime}\right)=\mathbb{E}\left[Q\left(\sqrt{\frac{\left|x_{1,1}^{\prime}-\hat{x}_{1,1}^{\prime}\right|^{2} \rho}{2}}\right)\right] \\
& \leq \mathbb{E}\left[\frac{1}{2} \exp \left(-\frac{\left|x_{1,1}^{\prime}-\hat{x}_{1,1}^{\prime}\right|^{2} \rho}{4}\right)\right] \leq \frac{1}{2}\left|\boldsymbol{I}_{2}+\frac{\left|x_{1,1}^{\prime}-\hat{x}_{1,1}^{\prime}\right|^{2} \rho}{4}\right|^{-1} \leq \frac{1}{2}\left|x_{1,1}^{\prime}-\hat{x}_{1,1}^{\prime}\right|\left(\frac{1}{4} \rho\right)^{-2}=\left|x_{1,1}^{\prime}-\hat{x}_{1,1}^{\prime}\right| \rho^{-2}
\end{aligned}
$$


where, $\mathbb{E}(\cdot)$ represents mathematical expectation. The value of $\left|x_{1,1}^{\prime}-\hat{x}_{1,1}^{\prime}\right|$ depends on the modulation mode.

In what follows, we derive $P_{e 1}$. According to the consistent bound method proposed by (12) in [12] and [13], $P_{e 1}$ satisfies the following equation

$$
\begin{aligned}
P_{e 1} & \leq \mathbb{E}\left[\sum_{\hat{\xi}} N(\xi, \hat{\xi}) P\left(\boldsymbol{x}_{0} \rightarrow \hat{\boldsymbol{x}}_{0}\right)\right] \\
& \leq \sum_{\xi=1}^{4} \sum_{q=1}^{M} \sum_{\hat{\xi}=1}^{4} \frac{N(\xi, \hat{\xi}) P\left(\boldsymbol{x}_{0} \rightarrow \hat{\boldsymbol{x}}_{0}\right)}{4 M}
\end{aligned}
$$

where, $M$ represents the modulation order, $\xi$ represents the serial number of transmit antennas, $\hat{\xi}$ is the number of antennas estimated by the base station. $\boldsymbol{x}_{0}$ is the first column of $\boldsymbol{X}_{1}$. $\hat{\boldsymbol{x}}_{0}$ is the decoding results obtained by decoding the first column of $\boldsymbol{X}_{1} \cdot N(\xi, \hat{\xi})$ represents the number of error bits between $\xi$ and $\hat{\xi}$.

It is known from (18) in [12] and (5) in [14],

$$
P\left(x_{0} \rightarrow \hat{\boldsymbol{x}}_{0}\right)=f(\alpha)^{N} \sum_{\beta=0}^{N-1}\left(\begin{array}{c}
N-1+\beta \\
\beta
\end{array}\right)(1-f(\alpha))^{\beta}
$$

where, $f(\alpha)=\frac{1}{2}\left(1-\sqrt{\frac{\alpha}{1+\alpha}}\right), \quad \alpha=\frac{1}{4 \sigma^{2}} \sum_{\xi=1}^{4}\left|x_{\xi}-\tilde{x}_{\xi}\right|^{2}, \quad x_{\xi}$ represent the $\xi$-th element of $x_{0}, \xi \in\{1,2,3,4\}$. Substitute (15) into (14) to get

$$
P_{e 1} \leq \sum_{\xi=1}^{4} \sum_{q=1}^{M} \sum_{\xi=1}^{4} \frac{N(\xi, \hat{\xi}) f(\alpha)^{N} \sum_{\beta=0}^{N-1}\left(\begin{array}{c}
N-1+\beta \\
\beta
\end{array}\right)(1-f(\alpha))^{\beta}}{4 M}
$$

Take (13) and (16) into (11) to get the SER of the system, as shown in (17).

$$
P_{e} \leq 1-\left(1-\sum_{\xi=1}^{4} \sum_{q=1}^{M} \sum_{\xi=1}^{4} \frac{N(\xi, \hat{\xi}) f(\alpha)^{N} \sum_{\beta=0}^{N-1}\left(\begin{array}{c}
N-1+\beta \\
\beta
\end{array}\right)(1-f(\alpha))^{\beta}}{4 M}\right)\left(1-\left|x_{1,1}^{\prime}-\hat{x}_{1,1}^{\prime}\right| \rho^{-2}\right)
$$

\section{Simulation Results and Analysis}

This chapter simulates the reliability of the proposed scheme and the reliability of [6]. Assume the channel follows the Rayleigh distribution and the noise is Gaussian white noise. There are 16 users using 4QAM modulation without channel coding.

The error probability $P_{e 1}$ of estimating the transmit antenna number when the base station is equipped with 64 antennas is shown is Figure 2. Simulation results show that when the SNR is $22 \mathrm{~dB}$, the error probability is about $10^{-3}$. Since the number of transmit antennas and the transmitted symbols are estimated simultaneously in [6], $P_{e 1}$ can't be obtained. So the error probability by estimating the transmit antenna number in [6] is not shown in Figure 2. It can be seen that when $N=128$, the $P_{e 1}$ is 0 while $\mathrm{SNR} \geq 0$. The reason is that the more the number of receiving antennas, the more accurate of the judgment. 
Therefore, the error probability of the estimate of transmit antenna number is not plot in Figure 2 when $N=128$.

The SER curves of the proposed scheme and the scheme in [6] with 64 antennas and 128 antennas is shown in Figure 3 and Figure 4 respectively. Simulation results show that when $N=64$, the reliability of the proposed scheme is lower than [6], when $N=128$, the reliability of the proposed scheme is better than [6]. That is because when $N=64$ the $P_{e 1}$ of the proposed scheme is higher, but when $N=128$ the $P_{e 1}$ of the proposed scheme is zero. From Figure 4, the gain of the proposed scheme is about $2.5 \mathrm{~dB}$ at the SER of $10^{-3}$ compared with the MP-GSM scheme, the gain is about $2.7 \mathrm{~dB}$ compared with the CHEMP-GSM scheme, and the gain is about $2.9 \mathrm{~dB}$ compared with the ML scheme.

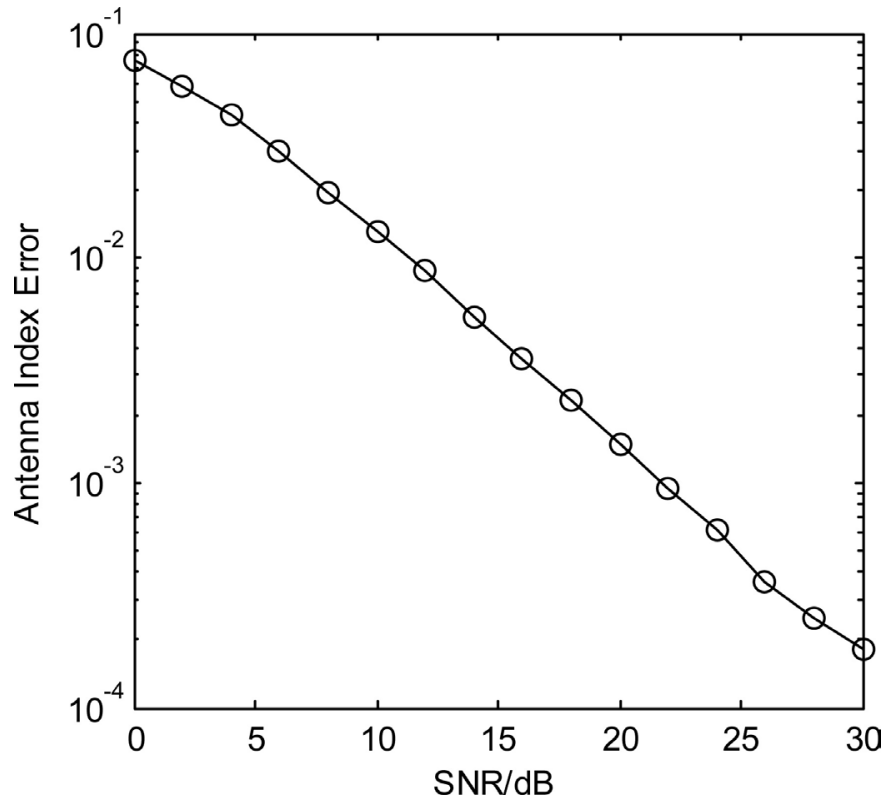

Figure 2. The error probability of estimating the transmit antenna with $N=64$.

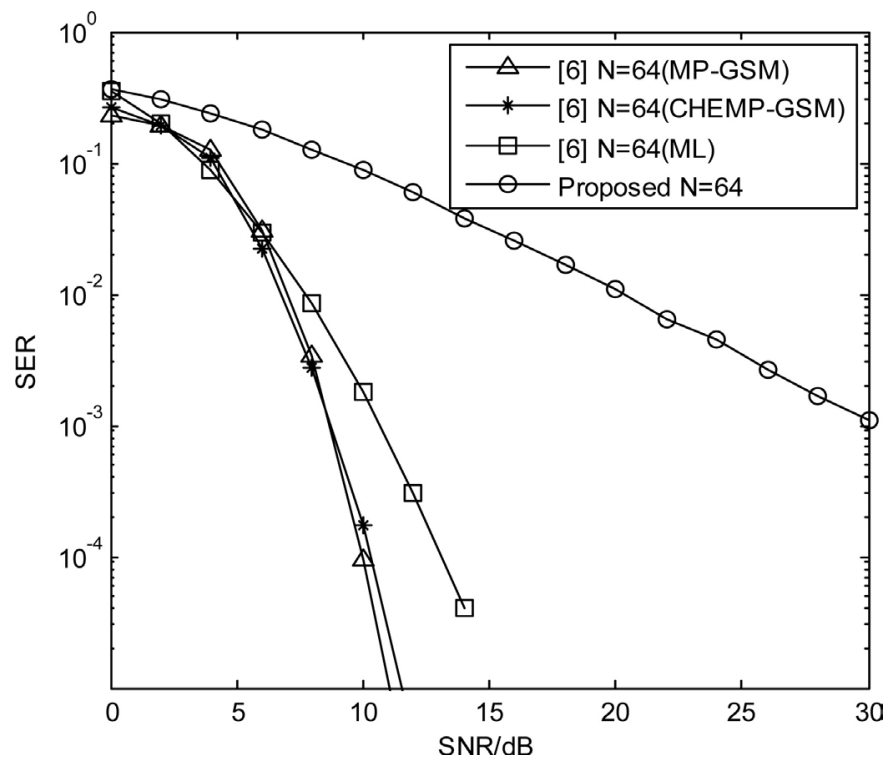

Figure 3. SER curves with $N=64$. 


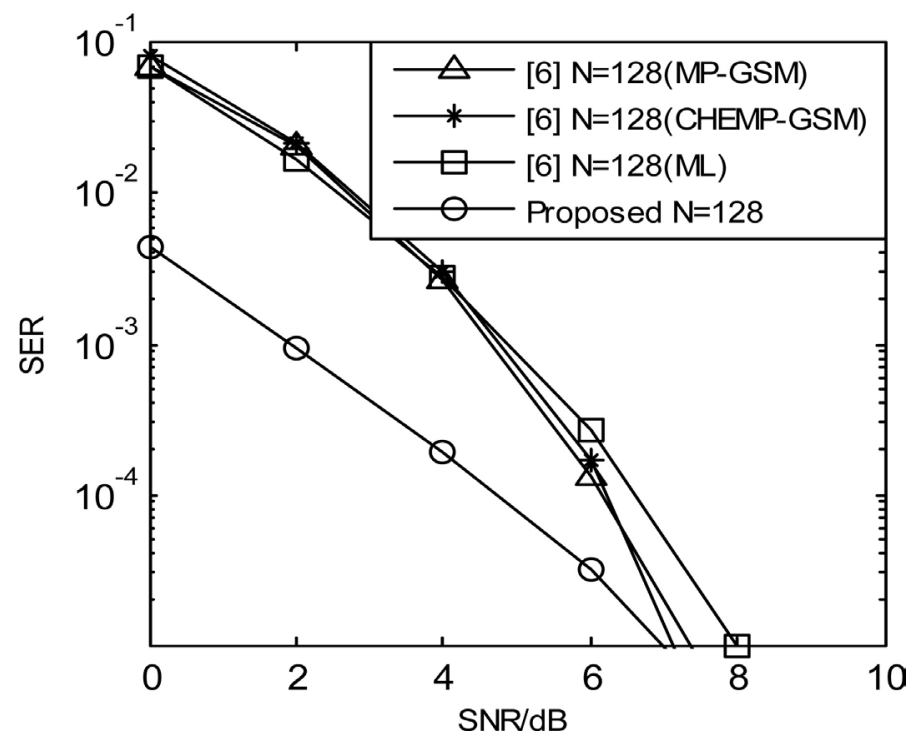

Figure 4. SER curves with $N=128$.

\section{Conclusion}

Compared with the existing scheme, each user adopts rate-2 space-time code. The transmission efficiency is the same as the existing scheme. The proposed scheme uses the orthogonal characteristics of the equivalent channel matrix corresponding to the Alamouti codeword to gradually separate each codeword, and then decodes each symbol of the codewords. The decoding complexity is greatly reduced. However, the reliability of the proposed scheme is greatly dependent on the estimated of the transmission antenna. How to reduce the dependency of the reliability of the system on the estimated transmit antenna number requires further study.

\section{References}

[1] Ye, Q., Bursalioglu, O.Y., Papadopoulos, H.C., et al. (2015) User Association and Interference Management in Massive MIMO HetNets. arXiv preprint arXiv:1509.07594,.

[2] Larsson, E., Edfors, O., Tufvesson, F., et al. (2014) Massive MIMO for Next Generation Wireless Systems. Communications Magazine, IEEE, 52, 186-195. https://doi.org/10.1109/MCOM.2014.6736761

[3] Zia, M.S. and Hassan, S.A. (2015) Outage Analysis of Multi-User Massive MIMO Systems Subject to Composite Fading. Vehicular Technology Conference (VTC Spring), 2015 IEEE 81st. IEEE, 1-5.

[4] Hassan, S.A. (2015) On Achievable Rates in Massive MIMO-Based Hexagonal Cellular System with Pilot Contamination. Vehicular Technology Conference (VTC Spring), 2015 IEEE 81st. IEEE, 1-5.

[5] Narasimhan, T.L. and Chockalingam, A. (2014) CHEMP Receiver for Large-Scale Multiuser MIMO Systems Using Spatial Modulation. Signal Processing Conference (EUSIPCO), 2014 Proceedings of the 22 nd European. IEEE, 86-90.

[6] Lakshmi Narasimhan, T., Raviteja, P. and Chockalingam, A. (2015) Generalized Spatial Modulation in Large-Scale Multiuser MIMO Systems. IEEE Transactions on 
Wireless Communications, 14, 3764-3779.

https://doi.org/10.1109/TWC.2015.2411651

[7] Tian, X.J. and Li, X.J. (2015) Improved Interference Cancellation Method over Y Channel. Journal of University of Science and Technology of China, 11, 943-948.

[8] Mesleh, R., Haas, H., Ahn, C.W., et al. (2006) Spatial Modulation-A New Low Complexity Spectral Efficiency Enhancing Technique. ChinaCom'06. First International Conference on Communications and Networking in China, 1-5.

[9] Kim, J., No, J. and Shin, D. (2014) Two-Way Relaying Schemes with Alamouti Code. 2014 International Conference on Information and Communication Technology Convergence (ICTC), 638-639.

[10] Kim, J., Jin, D.S., Jin, X., et al. (2015) Interference Alignment-and-Cancellation Scheme Based on Alamouti Code for the Three-User Multi-Input-Multi-Output Interference Channel. IET Communications, 9, 1278-1288.

https://doi.org/10.1049/iet-com.2014.1084

[11] Bhatnagar, M.R. and Hjørungnes, A. (2010) Improved Interference Cancellation Scheme for Two-User Detection of Alamouti Code. IEEE Transactions on Signal Processing, 58, 4459-4465. https://doi.org/10.1109/TSP.2010.2048211

[12] Naidoo, N.R., Xu, H.J. and Quazi, T.A.M. (2011) Spatial Modulation: Optimal Detector Asymptotic Performance and Multiple-Stage Detection. IET Communications, 5, 1368-1376. https://doi.org/10.1049/iet-com.2010.0667

[13] Proakis, J.G. (2009) Digital Communications. 5th Edition, McGraw-Hill, Singapore.

[14] Serafimovski, N., Sinanovic, S., Younis, A., et al. (2011) 2-User Multiple Access Spatial Modulation. IEEE GLOBECOM Workshops (GC Wkshps), 343-347.

Open Access Library

Submit or recommend next manuscript to OALib Journal and we will provide best service for you:

- Publication frequency: Monthly

- 9 subject areas of science, technology and medicine

- Fair and rigorous peer-review system

- Fast publication process

- Article promotion in various social networking sites (LinkedIn, Facebook, Twitter, etc.)

- Maximum dissemination of your research work

Submit Your Paper Online: Click Here to Submit

Or Contact service@oalib.com 